\title{
爆炸烧结 $\mathrm{Sm}_{2} \mathrm{Fe}_{17} \mathrm{~N}_{\mathrm{y}}$ 永磁体
}

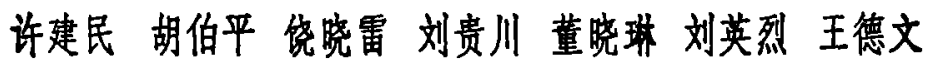 \\ 高景兰曹飞王亦忠宋琪刘整王晨西 \\ (中国科学院三环新材料研究开发公司，北京 100080)
}

\section{张登震蔡铰李世海}

（中国科学院力学研究所，北京 100080）(中国科学院物理研究所，北京 100080）

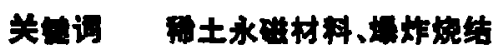

自从 1990 年初 Coey 和孙弘发现 $\mathrm{R}_{2} \mathrm{Fe}_{17} \mathrm{~N}$, 化合物以来 ${ }^{[1,2]}$, 人们对 $\mathrm{R}_{2} \mathrm{Fe}_{1} \mathrm{~N}$, 的内亲磁 性 ${ }^{(s-n)}$ 和永磁性 ${ }^{[s-11]}$ 进行了大量的研究。 $\mathrm{Sm}_{2} \mathrm{Fe}_{\mathrm{p}} \mathrm{N},(y>2)$ 由于具有优良的内寡磁性(居里

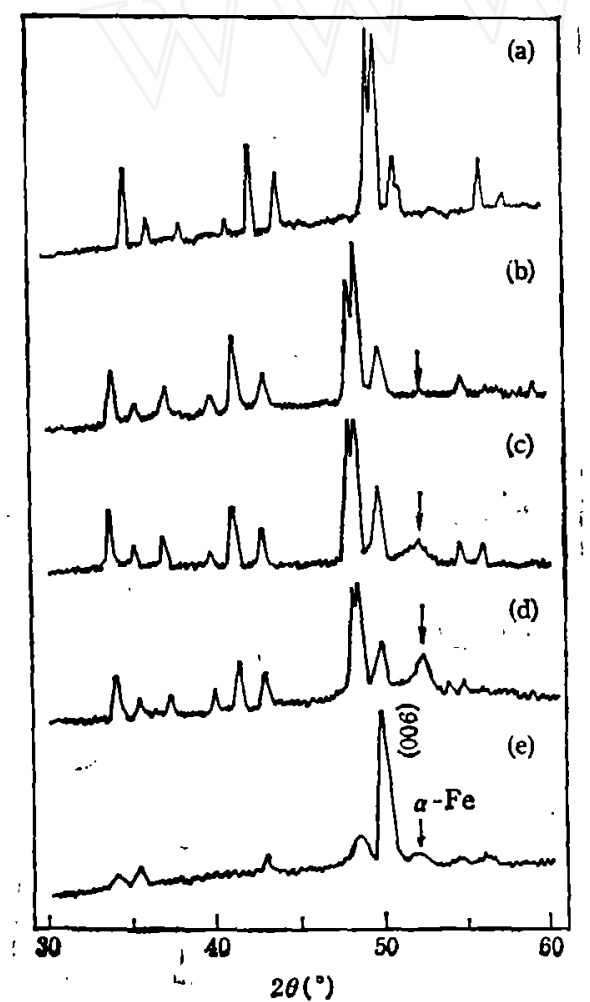

图 $1 \mathrm{x}$ 光衍射橧 (CoKa)

(a) $\mathrm{Sm}_{2} \mathrm{Fe}_{1}$, 合金, (b) $\mathrm{Sm}_{2} \mathrm{Fe}_{17} \mathrm{~N}$, 化合物,

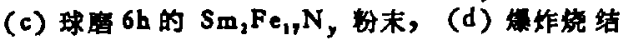

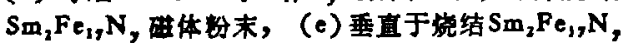
碰体取向端面

1992-02-09 收稿, 1992-04-01 收供改稆 温度 $749 \mathrm{~K}$ 和室温各向异性场 $14 \mathrm{~T}$ 大幅度高于 $\mathrm{Nd}_{2} \mathrm{Fe}_{14} \mathrm{~B}$ ，室温饱和磁化强度 $\left.1.5 \mathrm{~T}\right)$ ，故很有希望成 为新型稀土铁永磁体。采用机械合金化方法已获得

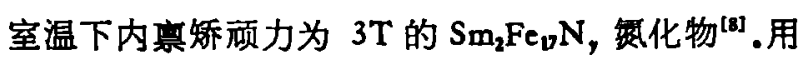
锌粉做粘结剂的 $\mathrm{Sm}_{2} \mathrm{Fe}_{v} \mathrm{~N}$, 磁体在室温下可达 $0.5 \mathrm{~T}$ 以上的内禀矫项力, 并且磁能积达 $80 \mathrm{~kJ} \cdot \mathrm{m}^{-3}$ 左 右 ${ }^{[0,103}$. 用环氧树脂做粘结剂制备的粘结磁体, 内 禀矫顾力为 $1.08 \mathrm{~T}$, 磁能积达 $72 \mathrm{~kJ} \cdot \mathrm{m}^{-3 \mathrm{dal}}$. 由于 $\mathrm{Sm}_{2} \mathrm{Fe}_{\mathrm{e}} \mathrm{N}$, 在高温下易分解, 故不能采用传统粉末冶 金工艺制备烧结磁体.为了克服这一困难，我们将爆 炸烧结技术用于 $\mathrm{Sm}_{2} \mathrm{Fe}_{12} \mathrm{~N}$, 的永磁体制备. 虽然爆 炸烧结方法曾用于单金属烧结 ${ }^{[23}$ 和铁体烧结 ${ }^{\text {[3] }}$, 但将该方法用于稀土永磁体制备在国内 外尚 无 报

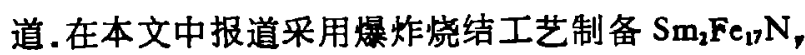
永磁体的研究结果。

$\mathrm{Sm}_{2} \mathrm{Fe}_{1}$ 合金由电弧妒熔炼，所有元素的纯度均 代于 $99.5 \%$ ，考虑到在熔炼过程中 $\mathrm{Sm}$ 的挥发 损 失,在配料时 $\mathrm{Sm}$ 的余量为 $15-40 \%$. 然后将合金. 在氩气保护下进行热处理,温度为 $950-1050^{\circ} \mathrm{C}$, 时 间为 5-10h. X 光衍射表明,合金为 $\mathrm{Th}_{2} \mathrm{Zn}_{1}$ 结构 单相. $\mathrm{Sm}_{2} \mathrm{Fe}_{1}$ 的渗氮特性研究参见我们已发表的 工作 ${ }^{[11]}$. 采用常规磁测量测得的 $\mathrm{Sm}_{3} \mathrm{Fe}_{1} \mathrm{~N}$, 内票磁 性同早期文嘀中的一致 ${ }^{[2,3,121}$. 
采用球磨工艺制备粒度小于 $3 \mu \mathrm{m}$ 的 $\mathrm{Sm}_{2} \mathrm{Fe}_{17} \mathrm{~N}$ ，磁粉，在约 $1.5 \mathrm{~T}$ 的外加磁场下取向压制 成型, 然后爆炸烧结, 制成 $\mathrm{Sm}_{2} \mathrm{Fe}_{17} \mathrm{~N}$, 永磁体. 烧结磁体的密度为 $(6.0-7.4) \times 10^{3} \mathrm{~kg} / \mathrm{m}^{3}$, 可 达理论密度的 $85 \%$ 以上. 下面我们所讨论的样品的密度为 $6.5 \times 10^{3} \mathrm{~kg} / \mathrm{m}^{3}$.

图 1 给出了几种不同情况下的 X 射线行射图. 可以清楚地看到, 爆炸烧结前后并不改变 $\mathrm{Sm}_{2} \mathrm{Fe}_{\mathrm{r}} \mathrm{N}$, 的结构(图 1(c) 和 (d)), 并且也不破坏预成型时的取向(图 1(e)). 图 1(b) 是贫 化后的 $\mathrm{Sm}_{2} \mathrm{Fe}_{17} \mathrm{~N}$, 化合物, 有微量的 $a-\mathrm{Fe}$ 相析出, 而在母合金中则几乎没有. 当球唐 $6 \mathrm{~h}$ 后 (图 I(c))， $\alpha-\mathrm{Fe}$ 相进一步增加. 而爆炸烧结后，磁体中 $\alpha-\mathrm{Fe}$ 相比爆炸烧结前的磁粉中 $\alpha-\mathrm{Fe}$ 相又有增加(图 1(d)).

图 2 是用提拉样品法在 $1.2 \mathrm{~T}$ 外磁场下测量的磁化强度随温度的变化. 从图中可以看到 主相 $\mathrm{Sm}_{2} \mathrm{Fe}_{17} \mathrm{~N}$, 的居里温度为 $475^{\circ} \mathrm{C}$, 在各种情况下均相同. 这也说明, 爆炸烧结并不影响 $\mathrm{Sm}_{2} \mathrm{Fe}_{\mathfrak{y}} \mathrm{N}$, 的内毫磁性. 曲线中的第二个台阶, 是 $\alpha-\mathrm{Fe}$ 相, 台阶的高低表明 $a-\mathrm{Fe}$ 含量的多 少,这同 X光衍射分析得到结论是一致的.

爆炸烧结 $\mathrm{Sm}_{2} \mathrm{Fe}_{17} \mathrm{~N}$ 磁体的退磁曲线如图 3(a) 所示, 剩余磁化强度 $B,-0.83 \mathrm{~T}$, 内亦 矫顽力 $\mu_{0} i H_{c}-0.57 \mathrm{~T}$, 最大磁能积 $(B H)_{\max }-88 \mathrm{kJm}^{-3}$. 同爆炸烧结前的磁粉取向粘结样 品相比 (图 3(b)), 烧结磁体的矩形度得到了一定的改善. 这可能是由于爆炸烧结, 磁粉互相 烧结相联而形成磁体, 使得它的磁畴结构与弥散粉末的磁畴结构相比发生了变化. 从图 3 中

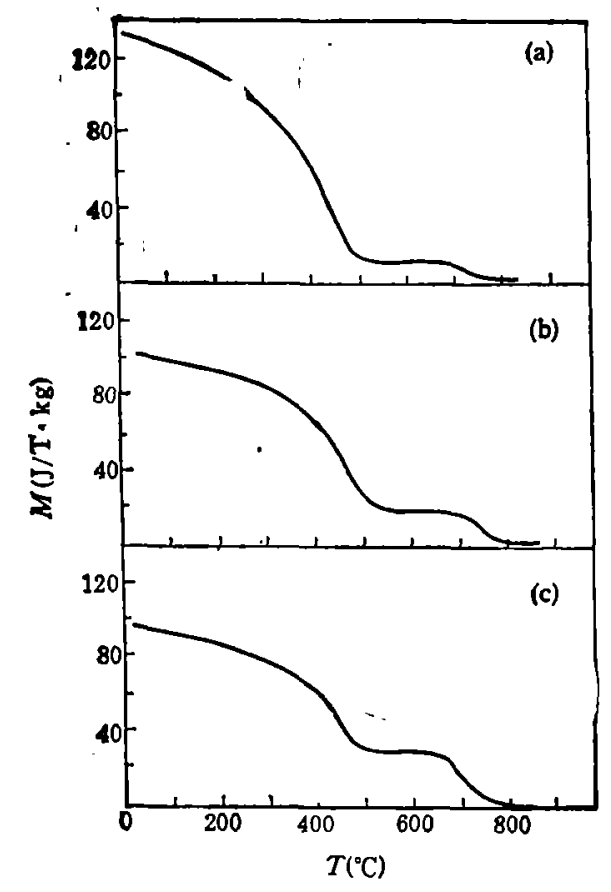

国2 化强度随温度的变化(外加磁场为 $1.2 \mathrm{~T}$ )

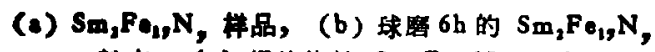

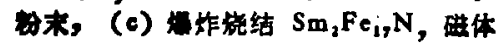

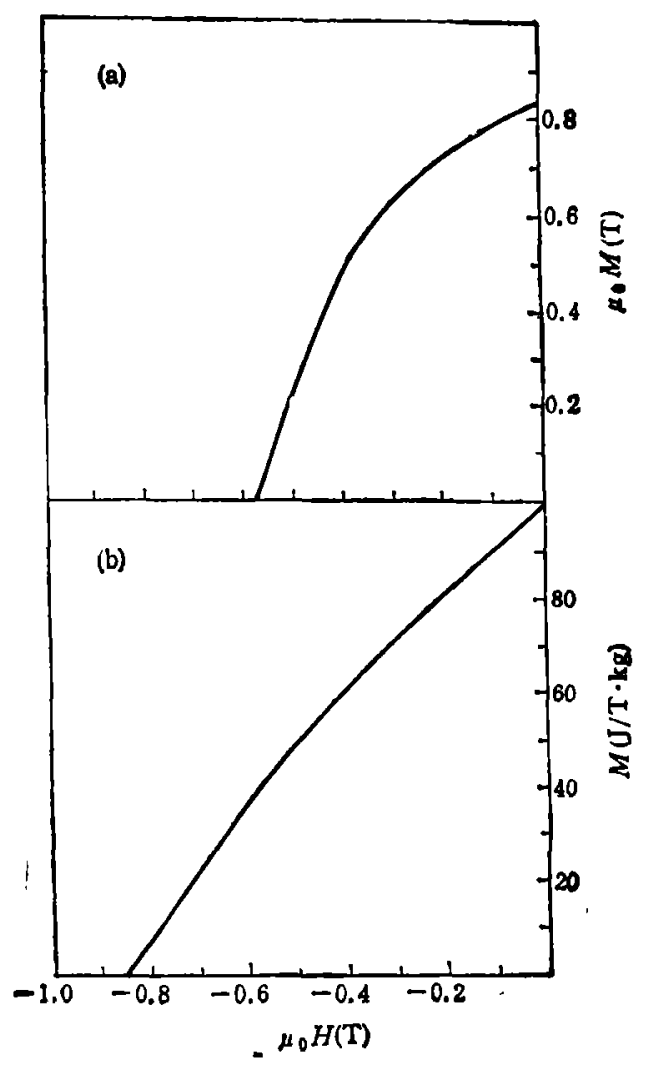

图 3 退磁曲线

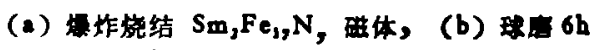
$\mathrm{Sm}_{2} \mathrm{Fe}_{1}, \mathrm{~N}$, 枮结样品 
还可以着到, 烧结磁体的矫颛力比爆炸烧结前降低了 (从 0.86T 降至 0.58T), 这说明爆炸过程 改变了磁的微结构. $\alpha-\mathrm{Fe}$ 相的增加也有可能影响磁体的矫顽力.

爆炸烧结作为一种新工艺，使磁粉在数波高压 $\left(\sim 10^{3} \mathrm{MPa}\right)$ 作用下瞬间 $\left(<10^{-\dot{6}_{s}}\right)$ 成型, 而不破坏 $\mathrm{Sm}_{2} \mathrm{Fe}_{2} \mathrm{~N}$, 氮化物的结构和磁性, 达到了常规高温烧结不能达到的目的. 与粘 结 $\mathrm{Sm}_{2} \mathrm{Fe}_{2} \mathrm{~N}$, 磁体相比 ${ }^{[11 \mathrm{U}}$, 爆炸烧结磁体改进了退磁曲线的矩形度，从而提高了磁能积。如果能 解进一步提高磁体密度,也就是提高剩余磁化强度, 进一步改善退磁线的矩形度,并保持足解 的矫顽力 $\left(\mu_{0} i H_{0}>0.5 \mathrm{~T}\right)$, 那么爆炸烧结 $\mathrm{Sm}_{2} \mathrm{Fe}_{\mathrm{v}} \mathrm{N}$, 磁体的磁能积还能进一步得到提高. 如, 设若 $\mathrm{Br}$ 为 $1 \mathrm{~T}$, 则理论最大磁能积达 $200 \mathrm{~kJ} \mathrm{~m}^{-3}$. 考虑到 $\mathrm{Sm}_{2} \mathrm{Fe}_{27} \mathrm{~N}$, 比 $\mathrm{Nd}_{2} \mathrm{Fe}_{14} \mathrm{~B}$ 的居 里温度高和瓷化物有较强的抗腐蚀特性, 所以 $\mathrm{Sm}_{2} \mathrm{Fe}_{\mathrm{v}} \mathrm{N}$, 烧结磁体可望在实际应用中占领一 席之地。

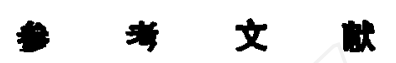

[ 1 ] Coey, J. M. D. and Sun Hong, J. Magn. Magn. Moser, 87(1990), L251.

[ 2 ] Sun Hong, Coey, J. M. D., Otani, Y. et 21., J. Phys. Conden. Mash, 2(1990), 6465.

[3] Hu Boping, Li Hongshuo, Sun Hong et al, J. Phys. Conden Mats, 3(1991), 3983.

[4] Buschow, R. H. J, Coehoorn, R., de Mooij, D. B. et al., J. Magn. Magn. Mazer, 92(1990), L35.

[ 5 ] Katter, M. Wecker, J., Schultz, L. et al, J. Magn. Magn. Moter, 92(1990), L14.

[ 6 ] Ibberion, R. M. Moze, O, Jacobs, T. H. et al, J. Phys., Conden. Mater, 3(1991), 1219.

[ 7 ] Liu, J. P, Bakker, K. et al., J. Lest-common Metals, 170(1991), 109.

[ 8 ] Schnittzke, K., Schultz, L., Wecker, J. et al., Appl. Phys. Lett, 57(1990), 2853

[9] Otani, Y., Moukarika, A., Sus Hong, et el., J. Appl. Phyr, 69(1991), 6735.

[10] Huang, M. Q., Zhang, L. Y, Ma, B. M. et al, J. Magn. Magn. Mazer, 102(1991), 91.

[11] 刘英烈、王福文、胡伯平等,科学通报, 36(1991), 24: 1850.

[12] Flinn, J. B, Williemson, R. L., Berry, R. A. et al., J. Appl. Phys., 64(1988), 1446.

[13] Atroschenko, E. S., Shock Weves for Industrial Applicasions (Ed, Murr, L. E.), chap. 5. 53 (in Rusuiaa), 1988, 1462-1465. 